\title{
Tetrahedron equation, Weyl group, AND QUANTUM DILOGARITHM
}

\author{
Andrei Bytsko And Alexander Volkov \\ Dedicated to Ludwig Faddeev on the occasion of his 80th birthday
}

\begin{abstract}
We derive a family of solutions to the tetrahedron equation using the RTT presentation of a two parametric quantized algebra of regular functions on an upper triangular subgroup of $G L(n)$. The key ingredients of the construction are the longest element of the Weyl group, the quantum dilogarithm function, and central elements of the quantized division algebra of rational functions on the subgroup in question.
\end{abstract}

\section{Introduction}

In what follows, $q, \lambda, \mu, \nu \in \mathbb{C}$ and $|q| \neq 1$. All algebras are considered over $\mathbb{C}$.

The quantum exponential function is the following formal power series in $x$ :

$$
\langle x\rangle_{q}=\sum_{n=0}^{\infty} \frac{(-x)^{n}}{(1-q) \ldots\left(1-q^{n}\right)} .
$$

$\langle x\rangle_{q}$ is the unique series in $\mathbb{C}[[x]]$ which satisfies the functional relation

$$
\langle q x\rangle_{q}=(1+x)\langle x\rangle_{q}
$$

and the condition $\langle 0\rangle_{q}=1$. The series inverse to $\langle x\rangle_{q}$ is given by

$$
1 /\langle x\rangle_{q}=\left\langle q^{-1} x\right\rangle_{q^{-1}}=\sum_{n=0}^{\infty} \frac{q^{\frac{n(n-1)}{2}} x^{n}}{(1-q) \ldots\left(1-q^{n}\right)} .
$$

Indeed, using (2), one verifies that $\theta(x) \equiv\langle x\rangle_{q}\left\langle q^{-1} x\right\rangle_{q^{-1}}$ has the property $\theta(q x)=\theta(x)$.

Quantum exponential function satisfies also the following identities [15, 5, 7]:

$$
\langle\mathrm{X}\rangle_{q}\langle\mathrm{Y}\rangle_{q}=\langle\mathrm{X}+\mathrm{Y}\rangle_{q}, \quad\langle\mathrm{X}\rangle_{q}\langle\mathrm{XY}\rangle_{q}\langle\mathrm{Y}\rangle_{q}=\langle\mathrm{Y}\rangle_{q}\langle\mathrm{X}\rangle_{q}
$$

involving two $q$-commuting indeterminates, $\mathrm{YX}=q \mathrm{XY}$. The second identity in (4) can be regarded as a quantum counterpart of the five term relation for the Rogers dilogarithm, see [5]. For this reason, $\langle x\rangle_{q}$ was named the quantum dilogarithm.

Using the quantum dilogarithm identity twice, one can derive another identity [9]:

$$
\langle\mathrm{X}\rangle_{q}\langle\mathrm{XY}\rangle_{q}\langle\mathrm{Z}\rangle_{q}\langle\mathrm{Y}\rangle_{q}=\langle\mathrm{Z}\rangle_{q}\langle\mathrm{ZX}\rangle_{q}\langle\mathrm{Y}\rangle_{q}\langle\mathrm{X}\rangle_{q}
$$

involving three pairwise $q$-commuting indeterminates,

$$
\mathrm{YX}=q \mathrm{XY}, \quad \mathrm{XZ}=q \mathrm{ZX}, \quad \mathrm{ZY}=q \mathrm{YZ} .
$$

Below we will need a more general statement. Namely, 
Lemma 1. Let indeterminates $\mathrm{X}, \mathrm{Y}, \mathrm{Z}$ satisfy relations (6) $)$. Let $S_{q, \lambda}(t), U_{q, \lambda}(t) \in \mathbb{C}[[t]]$ be non-constant formal power series in $t$ with coefficients depending on $q$ and $\lambda$. Then the following relations hold

$$
\begin{aligned}
S_{q, \lambda}(\mathrm{X}) S_{q, \lambda \mu}(\mathrm{XY}) S_{q, \mu}(\mathrm{Y}) & =S_{q, \mu}(\mathrm{Y}) S_{q, \lambda}(\mathrm{X}) \\
U_{q, \lambda}(\mathrm{X}) U_{q, \lambda \mu}(\mathrm{XY}) U_{q, \nu}(\mathrm{Z}) U_{q, \mu}(\mathrm{Y}) & =U_{q, \nu}(\mathrm{Z}) U_{q, \lambda \nu}(\mathrm{ZX}) U_{q, \mu}(\mathrm{Y}) U_{q, \lambda}(\mathrm{X})
\end{aligned}
$$

if and only if $S_{q, \lambda}(t)$ and $U_{q, \lambda}(t)$ are given by

$$
S_{q, \lambda}(t)=\left\langle q^{\frac{m(m-1)}{2}} \lambda^{k} t^{m}\right\rangle_{q^{m^{2}}}, \quad U_{q, \lambda}(t)=\kappa(q)\left\langle q^{\frac{m(m-1)}{2}} \lambda^{k} t^{m}\right\rangle_{q^{m^{2}}},
$$

where $m \in \mathbb{Z}_{+}, k \in \mathbb{Z}$, and $\kappa(q)$ is an arbitrary function of $q$.

Note that the order of the middle factors in (5) and (8) can be reversed because XY commutes with $\mathbf{Z}$ and likewise $\mathbf{Z X}$ commutes with $\mathrm{Y}$. Let us also remark that the commutative parameters $\lambda, \mu, \nu$ appearing in (7)-(8) can be set equal to unity by a suitable rescaling of $\mathrm{X}, \mathrm{Y}, \mathrm{Z}$. Therefore, these parameters are not of the same nature as the "physical" spectral parameters that appear, for instance, in the tetrahedron equation in [19]. However, we keep these parameters because equations which we consider remain nontrivial when some of these parameters are sent to zero. For instance, if $k$ in (9) is positive, equation (77) can be obtained from (8) by setting $\nu=0$ and taking into account that $\langle 0\rangle_{q}=1$.

The quantum exponential function often appears as a building block for solutions to the Yang-Baxter equation,

$$
R_{12} R_{13} R_{23}=R_{23} R_{13} R_{12} .
$$

See, for instance, Drinfeld's solution [3] for the R-matrix of $U_{q}\left(s l_{2}\right)$.

The Yang-Baxter equation has an interpretation in terms of three pairwise crossing straight lines in a plane. Its higher dimensional counterpart involving four pairwise intersecting planes in $\mathbb{R}^{3}$ is Zamolodchikov's tetrahedron equation [19]:

$$
R_{123} R_{145} R_{246} R_{356}=R_{356} R_{246} R_{145} R_{123} .
$$

The symbol $R$ in (10) and (11) can be assigned different meanings. For instance, it can be a set-theoretic map (and then the product of $R$ 's is understood as a composition) or a linear operator on a vector space. In the present article, we will regard $R$ as a function of non-commuting indeterminates.

In [8] (see also [10] for more details and [11] for some generalizations), a solution to the tetrahedron equation was constructed using the representation theory of $G L_{q}(n)$ - the quantized algebra of regular functions on the group $G L(n)$. Let us recall the scheme of this solution. Let $\pi$ be the irreducible representation of $G L_{q}(2)$ on an infinite-dimensional space $V$ constructed in [17]. The algebra homomorphisms $\varphi_{k}: G L_{q}(n) \rightarrow G L_{q}(2)$, where $k \in[1, n-1]$ labels a vertex of the $A_{n-1}$ Dynkin diagram, correspond to the embeddings $G L(2) \subset G L(n)$ of the classical groups. Let $s_{i_{1}} \cdots s_{i_{k}}$ be a reduced form of the longest element of the Weyl group for $G L(n)$. Then, as was shown in [18], $\pi_{i_{1}, \ldots, i_{k}}: x \rightarrow((\pi \circ$ $\left.\left.\varphi_{i_{1}}\right) \otimes \cdots \otimes\left(\pi \circ \varphi_{i_{k}}\right)\right) \circ(\Delta \otimes \cdots \otimes \Delta)(x)$ is an irreducible representation of $G L_{q}(n)$. Here $\Delta$ is the comultiplication of $G L_{q}(n)$. Let $S \in$ End $V^{\otimes 3}$ be the intertwiner of two irreducible representations of $G L_{q}(3): S \pi_{121}(x)=\pi_{212}(x) S$, and let $P_{13}$ denote the permutation of the first and the third tensor factors in $V^{\otimes 3}$. It was observed in 8 that $R=S \circ P_{13}$ is a solution to the tetrahedron equation because both sides of (11) for such $R$ are intertwiners of the 
representations $\pi_{121321}$ and $\pi_{323123}$ of $G L_{q}(4)$. Since these representations are irreducible, the intertwiners must coincide if they coincide on some vector from $V^{\otimes 6}$.

In the outlined above construction, the l.h.s. and the r.h.s. of (11) correspond to two ways of transforming the longest element of the Weyl group for $A_{3}$ with the help of the braid relations $s_{k} s_{k^{\prime}} s_{k}=s_{k^{\prime}} s_{k} s_{k^{\prime}}$ if $\left|k-k^{\prime}\right|=1$ and $s_{k} s_{k^{\prime}}=s_{k^{\prime}} s_{k}$ if $\left|k-k^{\prime}\right|>1$ :

$$
\begin{aligned}
& s_{1} s_{2} s_{1} s_{3} s_{2} s_{1}=s_{2} s_{1} s_{2} s_{3} s_{2} s_{1}=s_{2} s_{1} s_{3} s_{2} s_{3} s_{1}=s_{2} s_{3} s_{2} s_{1} s_{2} s_{3}=s_{3} s_{2} s_{1} s_{3} s_{2} s_{3}, \\
& s_{1} s_{2} s_{1} s_{3} s_{2} s_{1}=s_{1} s_{2} s_{3} s_{2} s_{1} s_{2}=s_{1} s_{3} s_{2} s_{3} s_{1} s_{2}=s_{3} s_{2} s_{1} s_{2} s_{3} s_{2}=s_{3} s_{2} s_{1} s_{3} s_{2} s_{3} .
\end{aligned}
$$

The aim of the present article is to construct solutions to (111) based on the same Weyl group structure but without utilising irreducible representations. For this purpose, we will first search for $R$ that transforms a $s_{1} s_{2} s_{1}$ product of non-commuting matrices into a $s_{2} s_{1} s_{2}$ product. This step is similar to finding the so-called fundamental solution to the YangBaxter equation by solving the RLL relation. An important difference of our approach from the above-mentioned construction using irreducible representations of $G L_{q}(n)$ is that we will deal with a division algebra whose center is not scalar. As a consequence, $R$ is not determined uniquely by the intertwining relation. Our first principal result is that the part of $R$ which is fixed by the intertwining relation is the quantum dilogarithm function of the image $w$ of a central element of the division algebra whereas the ambiguity factor in $R$ is a function of the dual image $\tilde{w}$ of the same central element (it turns out that $\tilde{w}$ and $w$ commute), cf. Theorem 1. Further, motivated by the obtained expression for $R$, we will search for a solution to (11) of the form $\tilde{R}=\mathrm{F} f(x) g(y)$, where $x$ and $y$ are monomials in the generators of a $q$-Weyl algebra and $F$ is a certain involution (a counterpart of the permutation in the Yang-Baxter equation) such that $\mathrm{F} w=\tilde{w} \mathrm{~F}$. Our second principal result is that both functions $f$ and $g$ are required to be the quantum dilogarithm function and $x, y$ must coincide with $w$ and $\tilde{w}$, cf. Theorem 2 , This implies, in particular, that $R$ found by solving the intertwining relation satisfies the tetrahedron equation if the ambiguity factor is also the quantum dilogarithm function. We observe that the latter condition holds automatically if $R$ is required to be an involution.

The proofs of all statements are given in the Appendix.

\section{An upper triangular quantum group}

In order to construct a family of solutions to the tetrahedron equation we will use (a certain quotient of) the two-parameter quantum group $G L_{q, p}(n)$. The following two-parameter generalization of the Jimbo-Drinfeld R-matrix is well known [14]:

$R_{q, p}^{(n)}=q p \sum_{i=1}^{n} E_{i i} \otimes E_{i i}+q \sum_{1 \leq i<j \leq n} E_{i i} \otimes E_{j j}+p \sum_{1 \leq j<i \leq n} E_{i i} \otimes E_{j j}+(q p-1) \sum_{1 \leq i<j \leq n} E_{i j} \otimes E_{j i}$,

where $E_{i j}$ are the basis matrices, i.e. $\left(E_{i j}\right)_{k l}=\delta_{i k} \delta_{j l}$. Matrix $R_{q, p}^{(n)}$ satisfies the Yang-Baxter equation (10). Matrix $\mathcal{P}^{(n)}=\sum_{i, j=1}^{n} E_{i j} \otimes E_{j i}$ acts on $\mathbb{C}^{n} \otimes \mathbb{C}^{n}$ as the permutation of the tensor factors and also provides a solution to (10).

Using the RTT approach [6], we define an associative algebra $G L_{q, p}^{+}(n)$ by the following matrix presentation:

$$
\check{R}_{q, p}^{(n)}(X(n) \otimes X(n))=(X(n) \otimes X(n)) \check{R}_{q, p}^{(n)},
$$


where $\check{R}_{q, p}^{(n)} \equiv \mathcal{P}^{(n)} R_{q, p}^{(n)}$, $\otimes$ stands for the Kronecker product, and $X(n)$ is an $n \times n$ upper triangular matrix with non-commuting entries, $x_{i j}, 1 \leq i \leq j \leq n$.

Explicitly, for every quadruple $\left(x_{i j}, x_{i k}, x_{m j}, x_{m k}\right)$ where $i<m \leq j<k$, eq. (14) is equivalent to the defining relations of $G L_{q, p}(2)$ :

$$
\begin{aligned}
& x_{i j} x_{i k}=q x_{i k} x_{i j}, \quad x_{m j} x_{m k}=q x_{m k} x_{m j}, \quad x_{i j} x_{m j}=p x_{m j} x_{i j}, \quad x_{i k} x_{m k}=p x_{m k} x_{i k}, \\
& x_{i k} x_{m j}=\frac{p}{q} x_{m j} x_{i k}, \quad x_{i j} x_{m k}-x_{m k} x_{i j}=\left(q-\frac{1}{p}\right) x_{i k} x_{m j}=\left(p-\frac{1}{q}\right) x_{m j} x_{i k} .
\end{aligned}
$$

For every triple $\left(x_{i j}, x_{i k}, x_{m k}\right)$, where $i<m, j<k, j<m$, eq. (14) is equivalent to

$$
x_{i j} x_{i k}=q x_{i k} x_{i j}, \quad x_{i k} x_{m k}=p x_{m k} x_{i k}, \quad x_{i j} x_{m k}=x_{m k} x_{i j},
$$

which is a reduction of (15) obtained by setting $x_{m j}$ to zero.

In what follows, we will consider a special case where $p$ is an integer power of $q$,

$$
p=q^{r}, \quad r \in \mathbb{Z}, \quad r \neq-1 .
$$

Lemma 2. If $p$ and $q$ are related as in (17), then the element

$$
C_{0}=\prod_{i=1}^{n}\left(x_{i i}\right)^{r^{n-i}}
$$

is central in $G L_{q, p}^{+}(n)$.

Let $S L_{q, q^{r}}^{+}(n)$ denote the quotient of $G L_{q, q^{r}}^{+}(n)$ by the ideal generated by $\left(C_{0}-1\right)$. We use the $S L$ notation for the resulting algebra since, in the $p=q$ case, $C_{0}$ coincides with the determinant of $X(n)$. Let us remark that $S L_{q, q^{r}}^{+}(n)$ can be equipped with the structure of a bialgebra by defining the comultiplication in the standard way, i.e., $\Delta(X(n))=X(n) \otimes$ $X(n)$. The element $C_{0}$ is group like w.r.t. this comultiplication.

Along with the algebra of regular functions $S L_{q, q^{r}}^{+}(n)$ generated by $x_{i j}$ we will consider the corresponding division algebra $D_{q, r}(n)$ of rational functions in non-commuting indeterminates $x_{i j}$ satisfying relations (15)-(16). Note that, in $D_{q, r}(n)$, the constraint $C_{0}=1$ can be resolved by expressing $x_{n n}$ in terms of other $x_{i i}$ which decreases the number of independent indeterminates by one. In particular, for $r=0$, we have $x_{n n}=C_{0}=1$.

In general, for $D_{q, r}(2)$, we have $x_{22}=x_{11}^{-r}$ and the only defining relations is

$$
x_{11} x_{12}=q x_{12} x_{11} .
$$

For $D_{q, r}(3)$, we have $x_{33}=x_{11}^{-r^{2}} x_{22}^{-r}$ along with the following set of relations for the independent indeterminates:

$$
\begin{aligned}
& x_{11} x_{12}=q x_{12} x_{11}, \quad x_{11} x_{13}=q x_{13} x_{11}, \quad x_{12} x_{13}=q x_{13} x_{12}, \quad x_{22} x_{23}=q x_{23} x_{22}, \\
& x_{13} x_{23}=q^{r} x_{23} x_{13}, \quad x_{12} x_{22}=q^{r} x_{22} x_{12}, \quad x_{13} x_{22}=q^{r-1} x_{22} x_{13}, \\
& x_{12} x_{23}-x_{23} x_{12}=\left(q-q^{-r}\right) x_{13} x_{22} .
\end{aligned}
$$

A remark is in order here. Let $S L_{r}^{+}(n)$ denote the subgroup of $n \times n$ upper triangular matrices with commuting entries satisfying the condition $C_{0}=1$, where $C_{0}$ is given by (18). The quasi-classical limit, $q=e^{\hbar}, p=e^{r \hbar}, \hbar \rightarrow 0$, of the RTT relations (14) for $X(n)$ induces the structure of a Poisson-Lie group on $S L_{r}^{+}(n)$. In particular, the classical counterpart 
of relation (19) is a log-canonical Poisson bracket: $\left\{x_{11}, x_{12}\right\}=x_{11} x_{12}$. For $n=3$, the Poisson structure corresponding to (20)-(22) is necessarily degenerate since it involves five variables whereas the dimension of the maximal symplectic leaf must be even and thus it is four. Therefore, the algebra $D_{q, r}(3)$ must have a non-trivial central element.

For $G L_{q}(n)$, the center of the corresponding division algebra was described in [2, 13. In the multi-parameter case, the structure of the center depends in general on the values of parameters [12]. However, for $D_{q, r}(3)$, it is not hard to find the center by a direct computation. Indeed, using (20)-(22), it is easy to check that the subalgebra of elements commuting with $x_{11}, x_{22}$, and $x_{13}$ is generated by $x_{11}^{r-1} x_{12} x_{23} x_{13}^{-1}$ and $x_{11}^{r-1} x_{22}$. Imposing the condition of commutativity with $x_{12}$ and $x_{23}$, we deduce that the center of $D_{q, r}(3)$ is generated by the element

$$
C_{1}=\left(x_{12} x_{23}-q x_{13} x_{22}\right) x_{11}^{-1} x_{33}^{-1} x_{13}^{-1}=\left(x_{12} x_{23}-q x_{13} x_{22}\right) x_{11}^{r^{2}-1} x_{22}^{r} x_{13}^{-1} .
$$

\section{Main construction}

Let $Q_{q}(n)$ denote the $q$-Weyl algebra with generators $a_{i}, a_{i}^{-1}, b_{i}, b_{i}^{-1}, i=1, \ldots, n$ and defining relations

$$
\begin{gathered}
a_{i} b_{j}=q^{\delta_{i j}} b_{j} a_{i}, \quad a_{i} a_{j}=a_{j} a_{i}, \quad b_{i} b_{j}=b_{j} b_{i}, \\
a_{i} a_{i}^{-1}=a_{i}^{-1} a_{i}=1, \quad b_{i} b_{i}^{-1}=b_{i}^{-1} b_{i}=1,
\end{gathered}
$$

where $\delta_{i j}$ stands for the Kronecker symbol.

Let us remark that $Q_{q}(n)$ admits a representation $\rho$ such that $\rho\left(a_{i}\right)=\exp \left(\alpha A_{i}\right)$ and $\rho\left(b_{i}\right)=\exp \left(\beta A_{i}^{+}\right), q=e^{\alpha \beta}$, where $A_{i}^{+}$and $A_{i}$ are the creation and annihilation operators on the Fock space for $n$ non-interacting particles, i.e. $\left[A_{i}, A_{j}^{+}\right]=1 \delta_{i j}$ and $\left[A_{i}^{+}, A_{j}^{+}\right]=\left[A_{i}, A_{j}\right]=0$. Replacing the quantum exponential function (1) with the modular quantum dilogarithm [4] which has no divergences at $|q|=1$, one can also consider a representation $\rho^{\prime}$ of $Q_{q}(n)$ where the generators are realised by unitary operators on the Hilbert space $L^{2}\left(\mathbb{R}^{n}\right): \rho^{\prime}\left(a_{i}\right)=\exp \left(\alpha \partial_{x_{i}}\right), \rho^{\prime}\left(b_{i}\right)=\exp \left(\sqrt{-1} \beta x_{i}\right), q=e^{\sqrt{-1}} \alpha \beta, \alpha, \beta \in \mathbb{R}$.

Let $\phi: D_{q, r}(2) \rightarrow Q_{q}(1)$ be the homomorphism such that $\phi\left(x_{11}\right)=a, \phi\left(x_{12}\right)=b$, $\phi\left(x_{22}\right)=a^{-r}$. It can be promoted to two homomorphisms $\phi^{(k)}: D_{q, r}(3) \rightarrow Q_{q}(1)$ that correspond to two embeddings $S L_{r}^{+}(2) \subset S L_{r}^{+}(3)$ of the classical groups, namely:

$$
\begin{aligned}
& \phi^{(1)}\left(x_{11}\right)=a, \quad \phi^{(1)}\left(x_{12}\right)=b, \quad \phi^{(1)}\left(x_{22}\right)=a^{-r}, \quad \phi^{(1)}\left(x_{k 3}\right)=\delta_{k 3}, \\
& \phi^{(2)}\left(x_{22}\right)=a, \quad \phi^{(2)}\left(x_{23}\right)=b, \quad \phi^{(2)}\left(x_{33}\right)=a^{-r}, \quad \phi^{(2)}\left(x_{1 k}\right)=\delta_{k 1} \text {. }
\end{aligned}
$$

Given $\lambda \in \mathbb{C}$, choose $\eta$ such that $\eta^{r^{2}+r+1}=\lambda^{-r}$. Consider two matrices:

$$
\begin{aligned}
& T=\eta\left(\begin{array}{ccc}
a_{1} & b_{1} & \\
& a_{1}^{-r} & \\
& & 1
\end{array}\right)\left(\begin{array}{ccc}
1 & & \\
& a_{2} & b_{2} \\
& & a_{2}^{-r}
\end{array}\right)\left(\begin{array}{ccc}
1 & & \\
& \lambda & \\
& & 1
\end{array}\right)\left(\begin{array}{ccc}
a_{3} & b_{3} & \\
& a_{3}^{-r} & \\
& & 1
\end{array}\right), \\
& \tilde{T}=\eta\left(\begin{array}{ccc}
1 & & \\
& a_{3} & b_{3} \\
& & a_{3}^{-r}
\end{array}\right)\left(\begin{array}{ccc}
1 & & \\
& \lambda & \\
& & 1
\end{array}\right)\left(\begin{array}{ccc}
a_{2} & b_{2} & \\
& a_{2}^{-r} & \\
& & 1
\end{array}\right)\left(\begin{array}{ccc}
1 & & \\
& a_{1} & b_{1} \\
& & a_{1}^{-r}
\end{array}\right) .
\end{aligned}
$$

Lemma 3. $T$ and $\tilde{T}$ satisfy relation (14) with $R$-matrix $R_{q, q^{r}}^{(3)}$. For $T$ and $\tilde{T}$, the value of $C_{0}$ given by (18) is equal to one. 
Thus, formulae (27) and (28) define (but not uniquely) homomorphisms $\phi^{(121)}, \phi^{(212)}$ : $D_{q, r}(3) \rightarrow Q_{q}(3)$ such that $T=\phi^{(121)}(X(3)), \tilde{T}=\phi^{(212)}(X(3))$. Under these homomorphisms, the images of the central element $C_{1}$ are monomials in $a_{i}$ and $b_{i}$. For us, it will be convenient to use

$$
\begin{aligned}
& w \equiv q \eta^{-1} \phi^{(121)}\left(C_{1}^{-1}\right)=a_{1}^{r} a_{2}^{-r} b_{1} b_{3}^{-1} a_{3} \\
& \tilde{w} \equiv q \eta^{-1} \phi^{(212)}\left(C_{1}^{-1}\right)=a_{3}^{-r} b_{1} b_{3}^{-1} a_{1}^{-1} a_{2} .
\end{aligned}
$$

Observe that

$$
\tilde{w}=\left(a_{1}^{-1} a_{2} a_{3}^{-1}\right)^{r+1} w=\eta \lambda^{-1} \phi^{(121)}\left(x_{11}^{-1} x_{22} x_{33}^{-1}\right) w=q \lambda^{-1} \phi^{(121)}\left(x_{11}^{-1} x_{22} x_{33}^{-1} C_{1}^{-1}\right) .
$$

Whence it follows that

$$
w \tilde{w}=\tilde{w} w .
$$

Now our aim is to construct solutions to the equation

$$
\mathrm{R}(\lambda) T=\tilde{T} \mathrm{R}(\lambda) .
$$

First, we consider an automorphism $\mathcal{R}$ of $Q_{q}(3)$ such that

$$
\mathcal{R}(T)=\tilde{T}
$$

where the action of $\mathcal{R}$ on the l.h.s. is entry-wise.

Proposition 1. (i) Equation (34) is equivalent to the following set of relations:

$$
\begin{aligned}
& \mathcal{R}\left(a_{1} a_{3}\right)=a_{2}, \quad \mathcal{R}\left(a_{2}\right)=a_{1} a_{3}, \quad \mathcal{R}\left(b_{1} b_{2}\right)=b_{1} b_{2}, \\
& \mathcal{R}\left(a_{1}^{-r} b_{2}\right)=a_{1}^{-r} b_{3}(1+\lambda w), \quad \mathcal{R}\left(a_{1} b_{3}\right)=(1+\lambda w)^{-1} a_{1} b_{2} .
\end{aligned}
$$

(ii) $\mathcal{R}$ acts on $w$ and $\tilde{w}$ as follows:

$$
\mathcal{R}(w)=\tilde{w}, \quad \mathcal{R}(\tilde{w})=w .
$$

Setting $\lambda=0$ in (35)-(36), we obtain another homomorphism. Clearly, since we have five relations (35)-(36) for six generators, this homomorphism is not defined uniquely.

Proposition 2. (i) If $\mathcal{F}$ is a homomorphism satisfying (35)-36) for $\lambda=0$ and $\mathcal{F}$ is an involution for all $r \neq-1$, then it is determined uniquely and is given by

$$
\begin{array}{lcc}
\mathcal{F}\left(a_{1}\right)=a_{1}, \quad \mathcal{F}\left(a_{2}\right)=a_{1} a_{3}, & \mathcal{F}\left(a_{3}\right)=a_{1}^{-1} a_{2}, \\
\mathcal{F}\left(b_{1}\right)=b_{1} b_{2} b_{3}^{-1}, & \mathcal{F}\left(b_{2}\right)=b_{3}, & \mathcal{F}\left(b_{3}\right)=b_{2} .
\end{array}
$$

(ii) $\mathcal{F}$ acts on $w$ and $\tilde{w}$ as follows:

$$
\mathcal{F}(w)=\tilde{w}, \quad \mathcal{F}(\tilde{w})=w .
$$

Let $\mathcal{F}_{i j k}, 1 \leq i<j<k \leq 6$, denote the automorphism of $Q_{q}(6)$ which acts nontrivially only on the $a$ 's and $b$ 's with labels $i, j, k$ and its action on these variables is given by (38)(39), where 1 is substituted by $i, 2$ by $j$, and 3 by $k$. It is known (see Proposition 2.1 in [9] and Lemma 2.13 in [1]) that $\mathcal{F}$ satisfies the tetrahedron equation,

$$
\mathcal{F}_{123} \circ \mathcal{F}_{145} \circ \mathcal{F}_{246} \circ \mathcal{F}_{356}=\mathcal{F}_{356} \circ \mathcal{F}_{246} \circ \mathcal{F}_{145} \circ \mathcal{F}_{123}
$$


This statement can be verified by computing the action of both sides of (41) on the monomial $M=a_{1}^{\alpha_{1}} b_{1}^{\beta_{1}} \ldots a_{6}^{\alpha_{6}} b_{6}^{\beta_{6}}$.

Thus, since $\mathcal{F}$ is an involution and a solution to the tetrahedron equation, it is a counterpart of the permutation $\mathcal{P}^{(n)}$ in the Yang-Baxter case. Therefore, by analogy with the Yang-Baxter case, one can look for solutions to (35)-(36) of the form $\mathcal{R}=\check{\mathcal{R}} \circ \mathcal{F}$. Using (35) -(36) and (38) - (39), we infer that $\check{\mathcal{R}}$ is an automorphism of $Q_{q}(3)$ such that

$$
\begin{aligned}
& \check{\mathcal{R}}\left(a_{1} a_{3}\right)=a_{1} a_{3}, \quad \check{\mathcal{R}}\left(a_{2}\right)=a_{2}, \quad \check{\mathcal{R}}\left(b_{1} b_{2}\right)=b_{1} b_{2}, \\
& \check{\mathcal{R}}\left(a_{1}^{-r} b_{3}\right)=a_{1}^{-r} b_{3}(1+\lambda w), \quad \check{\mathcal{R}}\left(a_{1} b_{2}\right)=(1+\lambda w)^{-1} a_{1} b_{2} .
\end{aligned}
$$

Note that $w$ and $\tilde{w}$ are invariant under the action of $\check{\mathcal{R}}$,

$$
\check{\mathcal{R}}(w)=w, \quad \check{\mathcal{R}}(\tilde{w})=\tilde{w} .
$$

Let us look for an invertible element $\check{\mathrm{R}}(\lambda) \in Q_{q}(3)$ such that

$$
\check{\mathrm{R}}(\lambda) x=\check{\mathcal{R}}(x) \check{\mathrm{R}}(\lambda)
$$

for every $x \in Q_{q}(3)$. From (42) and (44) it follows that $\check{\mathrm{R}}(\lambda)$ commutes with $a_{1} a_{3}, a_{2}, b_{1} b_{2}$, $w$, and $\tilde{w}$. It is not hard to check that the subalgebra in $Q_{q}(3)$ of elements commuting with this set is generated by $w$ and $\tilde{w}$. Therefore, $\check{R}(\lambda)$ is a function of $w$ and $\tilde{w}$ only.

Proposition 3. Every invertible $\check{\mathrm{R}}(\lambda) \in \mathbb{C}[[w, \tilde{w}]]$ which satisfies $(45)$ is given by

$$
\check{\mathrm{R}}(\lambda)=f_{q, \lambda}(\tilde{w})\langle\lambda w\rangle_{q^{r+1}},
$$

where $\langle t\rangle_{q}$ was defined in (11) and $f_{q, \lambda}(t)$ is an arbitrary invertible series from $\mathbb{C}[[t]]$.

Note that all $a_{i}$ and $b_{i} q$-commute with $w$ and $\tilde{w}$. Therefore, if $M$ is a monomial in $a_{i}$ and $b_{i}$, we have $\check{\mathcal{R}}(M)=M h_{1}(w) h_{2}(\tilde{w})$, where $h_{1}(t), h_{2}(t) \in \mathbb{C}[[t]]$.

Proposition 3 implies that we have constructed solutions to equation (33) of the following form:

$$
\mathrm{R}(\lambda)=f_{q, \lambda}(\tilde{w})\langle\lambda w\rangle_{q^{r+1}} \mathrm{~F}=\mathrm{F} f_{q, \lambda}(w)\langle\lambda \tilde{w}\rangle_{q^{r+1}},
$$

where the formal element $\mathrm{F}$ is an involution and $\mathrm{F} x=\mathcal{F}(x) \mathrm{F}$ for every $x \in Q_{q}(3)$. Note that the later relation cannot be resolved if $\mathrm{F}$ is assumed to be a formal power (or a formal Laurent) series in elements from $Q_{q}(3)$. Therefore, we will treat $\mathrm{F}$ as the generator of an outer automorphism.

Below we will need some generalization of (47) constructed by the same method. For this purpose, we consider a family of automorphisms $\psi_{s}, s \in \mathbb{Z}$, of $Q_{q}(3)$ defined on the generators as follows:

$$
\begin{array}{rrr}
\psi_{s}\left(a_{1}\right)=a_{1}, & \psi_{s}\left(a_{2}\right)=a_{2}, & \psi_{s}\left(a_{3}\right)=a_{3}, \\
\psi_{s}\left(b_{1}\right)=a_{2}^{s} a_{3}^{s} b_{1}, & \psi_{s}\left(b_{2}\right)=a_{1}^{s} b_{2}, & \psi_{s}\left(b_{3}\right)=a_{1}^{s} b_{3} .
\end{array}
$$

Clearly, we have $\psi_{s}^{-1}=\psi_{-s}$. It is straightforward to verify that $\psi_{s}$ commutes with $\mathcal{F}$ given by (38)-(39),

$$
\psi_{s} \circ \mathcal{F}=\mathcal{F} \circ \psi_{s}
$$

Consider $T_{s} \equiv \psi_{s}(T)$ and $\tilde{T}_{s} \equiv \psi_{s}(\tilde{T})$, that is matrices given by (27) and (28), where $b_{i}$ are replaced with $\psi_{s}\left(b_{i}\right)$. Since $\psi_{s}$ is an automorphism of $Q_{q}(3)$, Lemma 3 applies to $T_{s}$ 
and $\tilde{T}_{s}$ as well. Therefore, we can repeat the construction of this section in order to find solutions to the equation

$$
\mathrm{R}^{(s)}(\lambda) T_{s}=\tilde{T}_{s} \mathrm{R}^{(s)}(\lambda)
$$

of the form $\mathrm{R}^{(s)}(\lambda)=\check{\mathrm{R}}^{(s)}(\lambda) \mathrm{F}$, where $\mathrm{F}$ is the same as above. It is clear that $\mathcal{R}_{s} \equiv$ $\psi_{s} \circ \mathcal{R} \circ \psi_{-s}$ is an automorphism of $Q_{q}(3)$ such that $\mathcal{R}_{s}\left(T_{s}\right)=\tilde{T}_{s}$. Taking (50) into account, we deduce that $\mathcal{R}_{s}=\check{\mathcal{R}}_{s} \circ \mathcal{F}$, where $\check{\mathcal{R}}_{s}=\psi_{s} \circ \check{\mathcal{R}} \circ \psi_{-s}$. From the latter relation along with (45) and (46) we infer that $\check{\mathcal{R}}_{s}$ corresponds in the sense of Proposition 3 to $\psi_{s}(\check{\mathrm{R}}(\lambda))$. Therefore, we draw the following conclusion:

Theorem 1. Equation (51) has solutions of the following form:

$$
\mathrm{R}^{(s)}(\lambda)=f_{q, \lambda}\left(\tilde{w}^{(s)}\right)\left\langle\lambda w^{(s)}\right\rangle_{q^{r+1}} \mathrm{~F}=\mathrm{F} f_{q, \lambda}\left(w^{(s)}\right)\left\langle\lambda \tilde{w}^{(s)}\right\rangle_{q^{r+1}},
$$

where $f_{q, \lambda}(t)$ is an arbitrary invertible series from $\mathbb{C}[[t]]$ and

$$
w^{(s)} \equiv \psi_{s}(w)=a_{1}^{r-s} a_{2}^{s-r} b_{1} b_{3}^{-1} a_{3}^{s+1}, \quad \tilde{w}^{(s)} \equiv \psi_{s}(\tilde{w})=a_{3}^{s-r} b_{1} b_{3}^{-1} a_{1}^{-s-1} a_{2}^{s+1} .
$$

Note that setting $s=0$ in (152) we recover (47).

Applying the homomorphism $\psi_{s}$ to (32), we obtain

$$
w^{(s)} \tilde{w}^{(s)}=\tilde{w}^{(s)} w^{(s)} .
$$

In addition, it follows from (40) and (50) that $\mathrm{F} w^{(s)}=\tilde{w}^{(s)} \mathrm{F}$.

\section{The tetrahedron equation}

In order to discuss solutions to the tetrahedron equation we introduce in the standard way tensor copies of $\mathrm{R}^{(s)}(\lambda)$, namely

$$
\mathrm{R}_{i j k}^{(s)}(\lambda)=f_{q, \lambda}\left(\tilde{w}_{i j k}^{(s)}\right)\left\langle\lambda w_{i j k}^{(s)}\right\rangle_{q^{r+1}} \mathrm{~F}_{i j k}=\mathrm{F}_{i j k} f_{q, \lambda}\left(w_{i j k}^{(s)}\right)\left\langle\lambda \tilde{w}_{i j k}^{(s)}\right\rangle_{q^{r+1}}
$$

where $1 \leq i<j<k \leq 6$. Explicitly, the arguments in (55) are the following elements of $Q_{q}(6)$ :

$$
w_{i j k}^{(s)}=a_{i}^{r-s} a_{j}^{s-r} b_{i} b_{k}^{-1} a_{k}^{s+1}, \quad \tilde{w}_{i j k}^{(s)}=a_{k}^{s-r} b_{i} b_{k}^{-1} a_{i}^{-s-1} a_{j}^{s+1} .
$$

The key feature of equation (51) is that $\mathrm{R}^{(s)}(\lambda)$ is closely related to the transformation of the word $s_{1} s_{2} s_{1}$ into $s_{2} s_{1} s_{2}$. To make it explicit, let us rewrite (51) as follows:

$$
\mathrm{R}_{i j k}^{(s)}(\lambda) B_{i}^{(1)} B_{j}^{(2)} D_{\lambda}^{(12)} B_{k}^{(1)}=B_{k}^{(2)} D_{\lambda}^{(12)} B_{j}^{(1)} B_{i}^{(2)} \mathrm{R}_{i j k}^{(s)}(\lambda),
$$

where the notations are self-evident (we omit index $s$ in the matrix factors).

For the Weyl group of $A_{3}$, the two reduced forms of the longest element that are most distant from each other are $s_{1} s_{2} s_{1} s_{3} s_{2} s_{1}$ and $s_{3} s_{2} s_{1} s_{3} s_{2} s_{3}$. Therefore, we consider two $4 \times 4$

matrices with entries in $Q_{q}(6)$ corresponding to these words (we again omit index $s$ in the matrix factors):

$$
\begin{aligned}
& A_{s}=B_{1}^{(1)} B_{2}^{(2)} D_{\lambda}^{(12)} B_{3}^{(1)} B_{4}^{(3)} D_{\lambda \nu}^{(23)} B_{5}^{(2)} D_{\mu}^{(12)} B_{6}^{(1)}, \\
& \tilde{A}_{s}=B_{6}^{(3)} D_{\nu}^{(23)} B_{5}^{(2)} D_{\lambda \mu}^{(12)} B_{4}^{(1)} B_{3}^{(3)} D_{\lambda}^{(23)} B_{2}^{(2)} B_{1}^{(3)}
\end{aligned}
$$


Note that $B_{k}^{(1)}$ and $B_{j}^{(3)}$ commute.

Recall that the word $s_{1} s_{2} s_{1} s_{3} s_{2} s_{1}$ can be transformed into $s_{3} s_{2} s_{1} s_{3} s_{2} s_{3}$ along two different paths composed of four local steps, see (12). By (57), every local braid transformation $s_{l} s_{l+1} s_{l}=s_{l+1} s_{l} s_{l+1}$ in (12) corresponds to $\mathrm{R}_{i j k}^{(s)}(\lambda)$ for some $i, j, k$. Therefore, one can expect that $A_{s}$ is transformed into $\tilde{A}_{s}$ by products of four R's.

Proposition 4. (i) $A_{s}$ and $\tilde{A}_{s}$ satisfy the $R T T$ relation (14) with $R$-matrix $R_{q, q^{r}}^{(4)}$.

(ii) The following relations hold:

$$
\mathrm{M} A_{s}=\tilde{A}_{s} \mathrm{M}, \quad \mathrm{M}^{\prime} A_{s}=\tilde{A}_{s} \mathrm{M}^{\prime},
$$

where

$$
\mathrm{M}=\mathrm{R}_{123}^{(s)}(\lambda) \mathrm{R}_{145}^{(s)}(\lambda \mu) \mathrm{R}_{246}^{(s)}(\nu) \mathrm{R}_{356}^{(s)}(\mu), \quad \mathrm{M}^{\prime}=\mathrm{R}_{356}^{(s)}(\nu) \mathrm{R}_{246}^{(s)}(\mu) \mathrm{R}_{145}^{(s)}(\lambda \nu) \mathrm{R}_{123}^{(s)}(\lambda) .
$$

Part (i) of this Proposition implies that $A_{s}$ and $\tilde{A}_{s}$ (multiplied by suitable scalars) define two homomorphisms from $D_{q, r}(4)$ to $Q_{q}(6)$. However the center of $D_{q, r}(4)$ is not scalar. Therefore, relations (60) do not imply that $\mathrm{M}=\mathrm{M}^{\prime}$, which would mean that $\mathrm{R}^{(s)}(\lambda)$ satisfies the tetrahedron equation

$$
R_{123}(\lambda) R_{145}(\lambda \mu) R_{246}(\nu) R_{356}(\mu)=R_{356}(\nu) R_{246}(\mu) R_{145}(\lambda \nu) R_{123}(\lambda),
$$

but only that $\mathrm{M}^{-1} \mathrm{M}^{\prime}$ commutes with all the entries of $A_{s}$.

In order to find out whether $\mathrm{R}^{(s)}(\lambda)$ given by (52) satisfies the tetrahedron equation, we consider a more general ansatz.

Theorem 2. (i) Let $a_{i}, b_{i}, i=1, \ldots, 6$ satisfy relations 24) and $g_{q, \lambda}(t) \in \mathbb{C}[[t]]$ be a formal power series such that $g_{q, \lambda}(0)=1$. Then

$$
R_{123}(\lambda)=\mathrm{F}_{123} g_{q, \lambda}\left(q^{-\alpha_{1} \beta_{1}} a_{1}^{\alpha_{1}} a_{2}^{\alpha_{2}} a_{3}^{\alpha_{3}} b_{1}^{\beta_{1}} b_{2}^{\beta_{2}} b_{3}^{\beta_{3}}\right),
$$

where $\alpha_{i}, \beta_{i} \in \mathbb{Z}$, satisfies the tetrahedron equation (62) provided that

$$
\alpha_{1}+\alpha_{2}=0, \quad \beta_{2}=0, \quad \beta_{1}+\beta_{3}=0,
$$

and

$$
g_{q, \lambda}(t)=\left\langle Q^{\frac{m(m-1)}{2}} \lambda^{k} t^{m}\right\rangle_{Q^{m^{2}}},
$$

where $m \in \mathbb{Z}_{+}, k \in \mathbb{Z}$, and

$$
Q=q^{-\left(\alpha_{1}+\alpha_{3}\right) \beta_{1}} .
$$

(ii) Let $g_{q, \lambda}(t), f_{q, \lambda}(t) \in \mathbb{C}[[t]]$ be formal power series such that $g_{q, \lambda}(0)=f_{q, \lambda}(0)=1$. Then

$$
R_{123}(\lambda)=\mathrm{F}_{123} g_{q, \lambda}\left(q^{-\alpha \beta} a_{1}^{\alpha} a_{2}^{-\alpha} a_{3}^{\delta} b_{1}^{\beta} b_{3}^{-\beta}\right) f_{q, \lambda}\left(q^{-\hat{\alpha} \hat{\beta}} a_{1}^{\hat{\alpha}} a_{2}^{-\hat{\alpha}} a_{3}^{\hat{\delta}} b_{1}^{\hat{\beta}} b_{3}^{-\hat{\beta}}\right),
$$

where $\alpha, \beta, \delta, \hat{\alpha}, \hat{\beta}, \hat{\delta} \in \mathbb{Z}$, satisfies the tetrahedron equation (62) provided that

$$
\alpha \hat{\beta}+\hat{\delta} \beta=0, \quad \hat{\alpha} \beta+\delta \hat{\beta}=0,
$$

and

$$
g_{q, \lambda}(t)=\left\langle Q^{\frac{m(m-1)}{2}} \lambda^{k} t^{m}\right\rangle_{Q^{m^{2}}}, \quad f_{q, \lambda}(t)=\left\langle\widehat{Q}^{\frac{\widehat{m}(\widehat{m}-1)}{2}} \lambda^{l} t^{\widehat{m}}\right\rangle_{\widehat{Q}^{m^{2}}},
$$

where $m, \widehat{m} \in \mathbb{Z}_{+}, k, l \in \mathbb{Z}$, and

$$
Q=q^{-(\alpha+\delta) \beta}, \quad \hat{Q}=q^{-(\hat{\alpha}+\hat{\delta}) \hat{\beta}} .
$$


Let us remark that solutions to the tetrahedron equations of the type (63) were found earlier by various authors (usually for $\beta_{1}=m=1$ ). The case $\alpha_{1}=0, \alpha_{3}=-1$ was considered in [16], the case $\alpha_{1}=\alpha_{3}=-1$ in [9], the case $\alpha_{3}=-1-\alpha_{1}$ in [1].

It is a non-trivial fact that a solution of the type (63) admits the second factor, $f_{q, \lambda}$. Note that conditions (68) imply that the arguments of $g_{q, \lambda}$ and $f_{q, \lambda}$ in (67) commute. Furthermore, (68) and (70) imply that the corresponding $q$-parameters are related as follows:

$$
Q^{\widehat{\beta}^{2}}=\widehat{Q}^{-\beta^{2}} \text {. }
$$

Observe that the arguments of $g_{q, \lambda}$ and $f_{q, \lambda}$ in (67) coincide with $\tilde{w}^{(s)}$ and $q^{-(r+1)} w^{(s)}$, respectively, if we set

$$
\alpha=-\hat{\delta}=-s-1, \quad \hat{\alpha}=-\delta=r-s, \quad \beta=\hat{\beta}=1 .
$$

By (70), this corresponds to $Q=q^{r+1}$ and $\widehat{Q}=q^{-(r+1)}$. Therefore, Theorem 2 has the following corollaries concerning solutions to the intertwining relation (51):

Corollary 1. $\mathrm{R}^{(s)}(\lambda)$ given by (55) satisfies the tetrahedron equation (62) provided that invertible formal power series $f_{q, \lambda}(t) \in \mathbb{C}[[t]]$ either is constant or it is given by

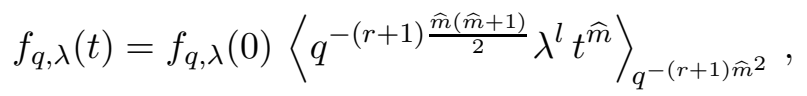

where $\widehat{m} \in \mathbb{Z}_{+}$and $l \in \mathbb{Z}$.

Corollary 2. If $\mathrm{R}^{(s)}(\lambda)$ given by (55) is an involution, i.e., $\left(\mathrm{R}^{(s)}(\lambda)\right)^{2}=1$, then it satisfies the tetrahedron equation (62).

Indeed, multiplying both expressions for $\mathrm{R}^{(s)}(\lambda)$ in (55) and taking into account that $\mathrm{F}^{2}=1$, we deduce that $\left(\mathrm{R}^{(s)}(\lambda)\right)^{2}=1$ holds provided that

$$
f_{q, \lambda}\left(w^{(s)}\right)\left\langle\lambda w^{(s)}\right\rangle_{q^{r+1}}=\left(f_{q, \lambda}\left(\tilde{w}^{(s)}\right)\left\langle\lambda \tilde{w}^{(s)}\right\rangle_{q^{r+1}}\right)^{-1} .
$$

Since $w^{(s)}$ and $\tilde{w}^{(s)}$ are algebraically independent, the formal power series on the l.h.s. and r.h.s. of (74) can coincide only if $f_{q, \lambda}(t)=f_{q, \lambda}(0) /\langle\lambda t\rangle_{q^{r+1}}$, where $f_{q, \lambda}(0)= \pm 1$. In this case, $\mathrm{R}^{(s)}(\lambda)$ is similar to $\mathrm{F}$

$$
\mathrm{R}^{(s)}(\lambda)= \pm\left\langle\lambda w^{(s)}\right\rangle_{q^{r+1}} \mathrm{~F} \frac{1}{\left\langle\lambda w^{(s)}\right\rangle_{q^{r+1}}}= \pm \frac{1}{\left\langle\lambda \tilde{w}^{(s)}\right\rangle_{q^{r+1}}} \mathrm{~F}\left\langle\lambda \tilde{w}^{(s)}\right\rangle_{q^{r+1}} .
$$

Note that, by (3), we have $f_{q, \lambda}(t)=f_{q, \lambda}(0) /\langle\lambda t\rangle_{q^{r+1}}=f_{q, \lambda}(0)\left\langle q^{-r-1} \lambda t\right\rangle_{q^{-(r+1)}}$ which coincides with (73) for $\hat{m}=l=1$. Therefore, by Corollary 1 , such $\mathrm{R}^{(s)}(\lambda)$ satisfies the tetrahedron equation.

\section{A Appendix}

Proof of Lemma 1. For the series $S_{q, \lambda}(t)=S_{0}(\lambda)+S_{1}(\lambda) t+\ldots$ (we omit the dependence on $q$ ), we have $S_{0}(\lambda) \neq 0$ (since otherwise the monomials of the least powers on the l.h.s. and on the r.h.s. of (7) do not match). Moreover, we have $S_{0}(\lambda) S_{0}(\lambda \mu) S_{0}(\mu)=S_{0}(\mu) S_{0}(\lambda)$, whence we conclude that $S_{0}(\lambda)=1$. Since relations (6) are homogeneous in $\mathrm{X}$ and $\mathrm{Y}$, the 
coefficients at $\mathrm{Y}^{k}$ on the l.h.s. and on the r.h.s. of (7) must match for every $k$. Let $m$ be the smallest positive integer such that $S_{m}(\lambda) \neq 0$. Matching the coefficients at $Y^{m}$ on the both sides of (17), we infer that

$$
S_{q, \lambda}(\mathrm{X})\left(S_{m}(\mu) \mathrm{Y}^{m}+S_{m}(\lambda \mu)(\mathrm{XY})^{m}\right)=S_{m}(\mu) \mathrm{Y}^{m} S_{q, \lambda}(\mathrm{X}) .
$$

Taking into account that $\mathrm{YX}=q \mathrm{XY}$, we can rewrite (76) as follows:

$$
S_{q, \lambda}(\mathrm{X})\left(1+q^{\frac{m(m-1)}{2}} \frac{S_{m}(\lambda \mu)}{S_{m}(\mu)} \mathbf{X}^{m}\right)=S_{q, \lambda}\left(q^{m} \mathbf{X}\right) .
$$

It follows that $\frac{S_{m}(\lambda \mu)}{S_{m}(\mu)}$ does not depend on $\mu$ and hence $S_{m}(\lambda) \sim \lambda^{k}, k \in \mathbb{Z}$. Thus, (77) acquires the form

$$
S_{q, \lambda}\left(q^{m} \mathbf{X}\right)=S_{q, \lambda}(\mathbf{X})\left(1+q^{\frac{m(m-1)}{2}} \lambda^{k} \mathbf{X}^{m}\right) .
$$

After a change of variables, $\hat{X}=q^{\frac{m(m-1)}{2}} \lambda^{k} X^{m}, \hat{q}=q^{m^{2}}$, functional equation (78) turns into equation (2). Whence we deduce that $S_{q, \lambda}(t)=\left\langle q^{m(m-1) / 2} \lambda^{k} t^{m}\right\rangle_{q^{m^{2}}}$.

By the same reasoning as above, we deduce that if the series $U_{q, \lambda}(t)=U_{0}(\lambda)+U_{1}(\lambda) t+\ldots$ is not vanishing, then $U_{0}(\lambda)$ does not vanish and does not depend on $\lambda$. Therefore, setting $\mathrm{Z}=0$ in (8), we conclude that $U_{q, \lambda}^{\prime}(t) \equiv U_{q, \lambda}(t) / U_{0}$ satisfies (7) and hence $U_{q, \lambda}(t)=$ $U_{0}\left\langle q^{m(m-1) / 2} \lambda^{k} t^{m}\right\rangle_{q^{m^{2}}}$ for some $m$ and $k$. In order to verify that relation (8) holds for such a series one has to make the same change of variables as above and compare the resulting relation with identity (5).

Proof of Lemma 2, Consider the element

$$
C_{0}=\prod_{i=1}^{n}\left(x_{i i}\right)^{\alpha_{i}},
$$

where $\alpha_{i}$ are unknown integers. The element $x_{j m}, j<m$, commutes with $x_{i i}$ non-trivially only if $i \in[j, m]$. Specifically, we have $x_{j m} x_{i i}=\theta x_{i i} x_{j m}$, where $\theta=q^{-1}$ if $i=j, \theta=q^{r}$ if $i=m$, and $\theta=q^{r-1}$ if $j<i<m$. Using these relations, we deduce that $x_{j m}$ commutes with $C_{0}$ provided that $\alpha$ 's satisfy the following relations for all pairs $j, m$, where $j<m$ :

$$
-\alpha_{j}+r \alpha_{m}+(r-1) \sum_{j<i<m} \alpha_{i}=0 .
$$

It is easy to verify that (80) holds if we choose $\alpha_{i}=r^{n-i}$.

Proof of Lemma 3. By the standard argument, if two matrices $X$ and $X^{\prime}$ satisfy (14) and entries of $X$ commute with these of $X^{\prime}$, then (14) holds for $X X^{\prime}$ as well. Every matrix factor in $T$ and $\tilde{T}$ is of the form $\phi(X(3))$, where $\phi$ is either one of the homomorphisms (25)-(26) or a trivial homomorphism sending $x_{i i}$ to $\lambda^{\delta_{i 2}}$ and vanishing on $x_{i j}$ if $i \neq j$. Therefore, every matrix factor in $T$ and $\tilde{T}$ satisfies relation (14) with $\check{R}_{q, q^{r}}^{(3)}$. Taking into account that entries of different factors in $T$ commute, we conclude that $T$ satisfies (14) with $\check{R}_{q, q^{r}}^{(3)}$. The same holds for $\tilde{T}$. The corresponding values of $C_{0}$ are straightforward to compute. 
Proof of Proposition 1, By a simple computation, we find

$$
\begin{aligned}
& T=\eta\left(\begin{array}{ccc}
a_{1} a_{3} & a_{1} b_{3}+\lambda b_{1} a_{2} a_{3}^{-r} & b_{1} b_{2} \\
& \lambda a_{1}^{-r} a_{2} a_{3}^{-r} & a_{1}^{-r} b_{2} \\
& & a_{2}^{-r}
\end{array}\right), \\
& \tilde{T}=\eta\left(\begin{array}{ccc}
a_{2} & a_{1} b_{2} & b_{1} b_{2} \\
& \lambda a_{1} a_{2}^{-r} a_{3} & b_{3} a_{1}^{-r}+\lambda b_{1} a_{2}^{-r} a_{3} \\
& & a_{1}^{-r} a_{3}^{-r}
\end{array}\right) .
\end{aligned}
$$

Now, comparing the diagonal entries and the upper right entries of $T$ and $\tilde{T}$, we obtain relations (35). Further, comparing the entries $(2,3)$ and taking formula (29) into account, we obtain the first relation in (36):

$$
\mathcal{R}\left(a_{1}^{-r} b_{2}\right)=b_{3} a_{1}^{-r}+\lambda b_{1} a_{2}^{-r} a_{3}=a_{1}^{-r} b_{3}(1+\lambda w) .
$$

Comparing the entries $(1,2)$, we infer that

$$
\mathcal{R}\left(a_{1} b_{3}\right)+\lambda \mathcal{R}\left(b_{1} a_{2} a_{3}^{-r}\right)=a_{1} b_{2} .
$$

Whence the second relation in (36) is derived as follows:

$$
\begin{aligned}
\mathcal{R}\left(a_{1} b_{3}\right) & =a_{1} b_{2}-\lambda \mathcal{R}\left(b_{1} b_{2}\right) \mathcal{R}\left(\left(a_{1}^{-r} b_{2}\right)^{-1}\right) \mathcal{R}\left(a_{1}^{-r} a_{3}^{-r}\right) \mathcal{R}\left(a_{2}\right) \\
& \stackrel{\text { 235, (83) }}{=} a_{1} b_{2}-\lambda b_{1} b_{2}(1+\lambda w)^{-1} a_{1}^{r} a_{2}^{-r} b_{3}^{-1} a_{3} a_{1} \\
& =b_{2} b_{1} b_{1}^{-1} a_{1}-\lambda b_{2} b_{1}(1+\lambda w)^{-1} w b_{1}^{-1} a_{1} \\
& =b_{2} b_{1}(1+\lambda w)^{-1} b_{1}^{-1} a_{1}=(1+\lambda w)^{-1} a_{1} b_{2} .
\end{aligned}
$$

In the last line we used that $w$ commutes with $b_{1} b_{2}$.

Consider the element $z \equiv a_{1}^{1-r} b_{2} b_{3}$. Multiplying relations in (36), we infer that

$$
\mathcal{R}(z)=z
$$

Note that $w$ and $\tilde{w}$ can be written as follows:

$$
w=q a_{2}^{-r} z^{-1}\left(b_{1} b_{2}\right)\left(a_{1} a_{3}\right), \quad \tilde{w}=q\left(a_{1} a_{3}\right)^{-r} z^{-1}\left(b_{1} b_{2}\right) a_{2} .
$$

Now, relations (37) are obvious if we take into account relations (35) and (85).

Proof of Proposition 2, Setting $\lambda=0$ in (35)-(36), we obtain

$$
\begin{gathered}
\mathcal{F}\left(a_{1} a_{3}\right)=a_{2}, \quad \mathcal{F}\left(a_{2}\right)=a_{1} a_{3}, \quad \mathcal{F}\left(b_{1} b_{2}\right)=b_{1} b_{2}, \\
\mathcal{F}\left(a_{1}^{-r} b_{2}\right)=a_{1}^{-r} b_{3}, \quad \mathcal{F}\left(a_{1} b_{3}\right)=a_{1} b_{2} .
\end{gathered}
$$

It follows from (88) that $\mathcal{F}(z)=z$. Therefore, formulae (86) and (87) imply that relations (40) hold (even if $\mathcal{F}$ is not an involution).

Consider $\mathcal{F}\left(a_{1}\right)$. Relations (87) $-(88)$ imply that $\mathcal{F}\left(a_{1}\right)$ commutes with $a_{2}, a_{1} a_{3}, a_{1}^{-r} b_{3}$, $a_{1} b_{2}$ and that $\mathcal{F}\left(a_{1}\right)\left(b_{1} b_{2}\right)=q\left(b_{1} b_{2}\right) \mathcal{F}\left(a_{1}\right)$. It is easy to check that every monomial satisfying these relations is of the form $a_{1}^{1-k} a_{2}^{k} a_{3}^{-r k} b_{1}^{k} b_{3}^{-k}, k \in \mathbb{Z}$. Comparing with (30), we infer that $\mathcal{F}\left(a_{1}\right)=a_{1} h(\tilde{w})$, where $h(t)$ is an unknown function. By (40), we have $\mathcal{F}(\tilde{w})=w$. 
Therefore, if $\mathcal{F}$ is an involution, we have $a_{1}=\mathcal{F}\left(\mathcal{F}\left(a_{1}\right)\right)=\mathcal{F}\left(a_{1} h(\tilde{w})\right)=a_{1} h(w) h(\tilde{w})$. Whence, $h(w) h(\tilde{w})=1$ which implies that $h(t)= \pm 1$ and $\mathcal{F}\left(a_{1}\right)= \pm a_{1}$. For the minus sign, we have $\mathcal{F}\left(\mathcal{F}\left(a_{1}^{-r} b_{2}\right)\right)=\mathcal{F}\left(a_{1}^{-r} b_{3}\right)=\mathcal{F}\left(a_{1}^{-r-1} a_{1} b_{3}\right)=(-1)^{-r-1} a_{1}^{-r} b_{2}$ and thus $\mathcal{F}$ is an involution only for odd $r$. Therefore, we have to choose the plus sign. Then, using $\mathcal{F}\left(a_{1}\right)=a_{1}$, it is straightforward to derive the rest of formulae in (38)-(39)).

Proof of Proposition 3. $\check{\mathrm{R}}(\lambda) \equiv \check{\mathrm{R}}_{\lambda}(w, \tilde{w})$ satisfies relations (42) automatically because $a_{1} a_{3}, a_{2}$, and $b_{1} b_{2}$ commute with both $w$ and $\tilde{w}$. Further, we note that $a_{1}^{-r} b_{3}$ and $a_{1} b_{2}$ commute with $\tilde{w}$ but have the following non-trivial relations with $w$ :

$$
w\left(a_{1}^{-r} b_{3}\right)=q^{r+1}\left(a_{1}^{-r} b_{3}\right) w, \quad w\left(a_{1} b_{2}\right)=q^{-r-1}\left(a_{1} b_{2}\right) w .
$$

Using these relations, it is not hard to verify that both equations (43) are equivalent to the following functional equation:

$$
\check{\mathrm{R}}_{\lambda}\left(q^{r+1} w, \tilde{w}\right)=(1+\lambda w) \check{\mathrm{R}}_{\lambda}(w, \tilde{w}) .
$$

This is the functional equation (2), where $q$ is replaced with $q^{r+1}$ and $\tilde{w}$ plays the role of a constant. Its solution in $\mathbb{C}[[w]]$ is unique up to a scalar multiple. The latter can be a non-trivial formal power series in $\tilde{w}$ whose coefficients can depend on $q$ and $\lambda$.

Proof of Proposition 4. Part (i). The same arguments apply as those that were used in the proof of Lemma 3 .

Part (ii). A verification is straightforward. For instance, we have

$$
\begin{aligned}
& \mathrm{M}^{\prime} A_{s}=R_{356}^{(s)}(\nu) R_{246}^{(s)}(\mu) R_{145}^{(s)}(\lambda \nu) R_{123}^{(s)}(\lambda) A_{s} \\
& =R_{356}^{(s)}(\nu) R_{246}^{(s)}(\mu) R_{145}^{(s)}(\lambda \nu) R_{123}^{(s)}(\lambda) \frac{B_{1}^{(1)} B_{2}^{(2)} D_{\lambda}^{(12)} B_{3}^{(1)} B_{4}^{(3)} D_{\lambda \nu}^{(23)} B_{5}^{(2)} D_{\mu}^{(12)} B_{6}^{(1)}}{=} R_{356}^{(s)}(\nu) R_{246}^{(s)}(\mu) R_{145}^{(s)}(\lambda \nu) B_{3}^{(2)} D_{\lambda}^{(12)} B_{2}^{(1)} \frac{B_{1}^{(2)} B_{4}^{(3)} D_{\lambda \nu}^{(23)} B_{5}^{(2)} D_{\mu}^{(12)} B_{6}^{(1)} R_{123}^{(s)}(\lambda)}{=} R_{356}^{(s)}(\nu) R_{246}^{(s)}(\mu) B_{3}^{(2)} D_{\lambda}^{(12)} B_{2}^{(1)} B_{5}^{(3)} D_{\lambda \nu}^{(23)} B_{4}^{(2)} B_{1}^{(3)} D_{\mu}^{(12)} B_{6}^{(1)} R_{145}^{(s)}(\lambda \nu) R_{123}^{(s)}(\lambda) \\
& =R_{356}^{(s)}(\nu) R_{246}^{(s)}(\mu) B_{3}^{(2)} B_{5}^{(3)} D_{\nu}^{(23)} D_{\lambda}^{(12)} D_{\lambda}^{(23)} \frac{B_{2}^{(1)} B_{4}^{(2)} D_{\mu}^{(12)} B_{6}^{(1)} B_{1}^{(3)} R_{145}^{(s)}(\lambda \nu) R_{123}^{(s)}(\lambda)}{=} R_{356}^{(s)}(\nu) B_{3}^{(2)} B_{5}^{(3)} D_{\nu}^{(23)} D_{\lambda}^{(12)} D_{\lambda}^{(23)} B_{6}^{(2)} D_{\mu}^{(12)} B_{4}^{(1)} B_{2}^{(2)} B_{1}^{(3)} R_{246}^{(s)}(\mu) R_{145}^{(s)}(\lambda \nu) R_{123}^{(s)}(\lambda) \\
& =R_{356}^{(s)}(\nu) B_{3}^{(2)} B_{5}^{(3)} D_{\nu}^{(23)} B_{6}^{(2)} D_{\lambda \mu}^{(12)} B_{4}^{(1)} D_{\lambda}^{(23)} B_{2}^{(2)} B_{1}^{(3)} R_{246}^{(s)}(\mu) R_{145}^{(s)}(\lambda \nu) R_{123}^{(s)}(\lambda) \\
& =B_{6}^{(3)} D_{\nu}^{(23)} B_{5}^{(2)} B_{3}^{(3)} D_{\lambda \mu}^{(12)} B_{4}^{(1)} D_{\lambda}^{(23)} B_{2}^{(2)} B_{1}^{(3)} R_{356}^{(s)}(\nu) R_{246}^{(s)}(\mu) R_{145}^{(s)}(\lambda \nu) R_{123}^{(s)}(\lambda) \\
& =\tilde{A}_{s} R_{356}^{(s)}(\nu) R_{246}^{(s)}(\mu) R_{145}^{(s)}(\lambda \nu) R_{123}^{(s)}(\lambda)=\tilde{A}_{s} \mathrm{M}^{\prime} .
\end{aligned}
$$

The underlined terms were transformed by applying relation (57). The remaining transformations are simply permutations of commuting terms.

Proof of Theorem 2. Part (i). Substitute (63) into (62) and pull all F's to the left transforming the arguments of $g$ 's according to (38) and (39). The products of F's can be cancelled due to (41) and we are left with a product of four $g$ 's on the each side,

$$
g_{q, \lambda}(\mathbf{X}) g_{q, \lambda \mu}(\mathbf{T}) g_{q, \nu}(\mathbf{Z}) g_{q, \mu}(\mathbf{Y})=g_{q, \nu}\left(\mathbf{Z}^{\prime}\right) g_{q, \mu}\left(\mathrm{Y}^{\prime}\right) g_{q, \lambda \nu}\left(\mathbf{T}^{\prime}\right) g_{q, \lambda}\left(\mathbf{X}^{\prime}\right)
$$

where the arguments of $g$ 's are monomials in $a_{i}$ 's and $b_{i}$ 's, for instance,

$$
\mathrm{X}=a_{1}^{\alpha_{1}} a_{2}^{\alpha_{2}} a_{3}^{\alpha_{3}} b_{1}^{\beta_{1}} b_{2}^{\beta_{2}} b_{3}^{\beta_{3}} b_{4}^{\beta_{2}} b_{5}^{\beta_{3}+\beta_{1}-\beta_{2}} b_{6}^{-\beta_{1}-\beta_{3}}, \quad \mathrm{Y}^{\prime}=a_{1}^{\alpha_{1}+\alpha_{2}} a_{3}^{\alpha_{1}} a_{5}^{\alpha_{2}} a_{6}^{\alpha_{3}} b_{2}^{\beta_{1}} b_{4}^{\beta_{2}} b_{6}^{\beta_{3}} .
$$


If conditions (64) hold, then we have $X=X^{\prime}, Y=Y^{\prime}, Z=Z^{\prime}, T=X Y, T^{\prime}=Z X$ and, moreover, $\mathrm{X}, \mathrm{Y}, \mathrm{Z}$ satisfy relations (6) with $q$ replaced by $Q$ given by (66). Therefore, by Lemma 1, $g_{q, \lambda}(t)$ must be of the form (65).

Part (ii). Substitute (67) into (62), pull all F's to the left transforming the arguments of $g$ 's and f's according to (38) and (39), and then cancel the products of F's. Taking into account that the arguments of $g$ and $f$ in (67) satisfy conditions (64), we obtain the following equality

$$
\begin{aligned}
& g_{q, \lambda}(\mathrm{X}) f_{q, \lambda}(\tilde{\mathrm{X}}) g_{q, \lambda \mu}(\mathrm{XY}) f_{q, \lambda \mu}(\tilde{\mathbf{X}} \tilde{\mathbf{Y}}) g_{q, \nu}(\mathrm{Z}) f_{q, \nu}(\tilde{\mathbf{Z}}) g_{q, \mu}(\mathrm{Y}) f_{q, \mu}(\tilde{\mathbf{Y}}) \\
& =g_{q, \nu}(\mathbf{Z}) f_{q, \nu}(\tilde{\mathbf{Z}}) g_{q, \mu}(\mathbf{Y}) f_{q, \mu}(\tilde{\mathbf{Y}}) g_{q, \lambda \nu}(\mathbf{Z X}) f_{q, \lambda \nu}(\tilde{\mathbf{Z}} \tilde{\mathbf{X}}) g_{q, \lambda}(\mathbf{X}) f_{q, \lambda}(\tilde{\mathbf{X}})
\end{aligned}
$$

where both triples $\mathrm{X}, \mathrm{Y}, \mathrm{Z}$, and $\tilde{X}, \tilde{\mathrm{Y}}, \tilde{\mathrm{Z}}$ satisfy relations (6) with $q$ replaced, respectively, by $Q$ and $\widehat{Q}$ given by (70). Furthermore, if conditions (68) hold, then every element of the triple $X, Y, Z$ commutes with every element of the triple $\tilde{X}, \tilde{Y}, \tilde{Z}$. Therefore, equality (93) holds if its $g$ and $f$ parts satisfy separately relation (8). By Lemma 1, this requires $g_{q, \lambda}(t)$ and $f_{q, \lambda}(t)$ to be of the form (69).

Acknowledgements. We thank R. Kashaev and V. Tarasov for useful remarks. This work was supported by the European Research Council (ERC) grant MODFLAT, by the Swiss National Science Foundation grant 200020-141329, and in part by the Russian Fund for Basic Research grants 14-01-00341 and 13-01-12405-ofi-m.

\section{References}

[1] A. Bytsko and A. Volkov, Tetrahedron equation and cyclic quantum dilogarithm identities.arXiv:1304.1641, Int. Math. Res. Notices (to appear).

[2] G. Cliff, The division ring of quotients of the coordinate ring of the quantum general linear group. - J. London Math. Soc. (2) 51 (1995) no. 3, 503-513.

[3] V.G. Drinfeld, Quantum groups.- J. Soviet Math. 41 (1988) no. 2, 898-915.

[4] L.D. Faddeev, Discrete Heisenberg-Weyl group and modular group. - Lett. Math. Phys. 34 (1995) no. 3, 249-254.

[5] L.D. Faddeev and R.M. Kashaev, Quantum dilogarithm. - Modern Physics Letters A9 (1994) no. $5,427-434$.

[6] L.D. Faddeev, N.Yu. Reshetikhin, and L.A. Takhtadzhyan, Quantization of Lie groups and Lie algebras. - Leningrad Math. J. 1 (1990) no. 1, 193-225.

[7] L.D. Faddeev and A.Yu. Volkov, Abelian current algebra and the Virasoro algebra on the lattice.- Physics Letters B315 (1993) no. 3-4, 311-318.

[8] M. Kapranov and V. Voevodsky, 2-categories and Zamolodchikov tetrahedra equations. In: Algebraic groups and their generalizations: quantum and infinite-dimensional methods, 177-259. Proc. Sympos. Pure Math., 56, Part 2. AMS, 1994.

[9] R.M. Kashaev and A.Yu. Volkov, From the tetrahedron equation to universal R-matrices. In: L.D. Faddeev's Seminar on Mathematical Physics, 79-89. AMS Transl. Ser. 2, 201. AMS, 2000.

[10] D. Kazhdan and Ya. Soibelman, Representations of the quantized function algebras, 2-categories and Zamolodchikov tetrahedra equation. In: The Gel'fand Mathematical Seminars, 1990-1992, 163-171. Birkhäuser, 1993.

[11] A. Kuniba and M. Okado, Tetrahedron and 3D reflection equations from quantized algebra of functions. - J. Phys. A45 (2012) no. 46, 465206. 
[12] V.G. Mosin and A.N. Panov, Quotient skew fields and central elements of multiparametric quantizations. - Sbornik. Mathematics 187 (1996) no. 6, 835-855.

[13] A.N. Panov, Skew fields of twisted rational functions and the skew field of rational functions on $G L_{q}(n, K)$. - St. Petersburg Math. J. 7 (1996) no. 1, 129-143.

[14] N. Reshetikhin, Multiparameter quantum groups and twisted quasitriangular Hopf algebras.Lett. Math. Phys. 20 (1990) no. 4, 331-335.

[15] M.P. Schützenberger, Une interprétation de certaines solutions de l'équation fonctionnelle: $F(x+y)=F(x) F(y)$. - C. R. Acad. Sci. Paris 236 (1953) 352-353.

[16] S.M. Sergeev, Two-dimensional $R$-matrices - descendants of three-dimensional $R$ matrices. - Modern Physics Letters A12 (1997) no. 19, 1393-1410.

[17] Ya.S. Soibelman and L.L. Vaksman, An algebra of functions on the quantum group $S U(2)$.Funct. Anal. Appl. 22 (1989) no. 3, 170-181.

[18] Ya.S. Soibelman and L.L. Vaksman, Algebra of functions on the quantum group $S U(n+1)$, and odd-dimensional quantum spheres. - Leningrad Math. J. 2 (1991) no. 5, 1023-1042.

[19] A.B. Zamolodchikov, Tetrahedron equations and the relativistic S-matrix of straight-strings in 2+1-dimensions. - Commun. Math. Phys. 79 (1981) no. 4, 489-505.

Section of Mathematics, University of Geneva, C.P. 64, 1211 Genève 4, Switzerland Steklov Mathematical Institute, Russian Academy of Sciences, Fontanka 27, 191023, St. Petersburg, Russia 